\title{
Investigation of the Therapeutic Effects of Chloroquine in Adriamycin-Induced Hepatotoxicity
}

\author{
Ali Tuğrul Akin ${ }^{{ }^{*}}$, Emin Kaymak ${ }^{2}$, Emel Öztürk ${ }^{3}$, Derya Karabulut ${ }^{4}$, Nurhan Kuloğlư ${ }^{5}$, Tayfun Ceylan ${ }^{6}$, \\ Ayşe Toluk ${ }^{1}$
}

\begin{abstract}
The aim of this study is to investigate the therapeutic effects of Chloroquine (CLQ) against Adriamycin (ADR) induced hepatotoxicity. ADR is a chemotherapeutic agent used in the treatment of many cancer types, but it causes hepatotoxicity. CLQ is used as an anti-inflammatory drug in the treatment of malaria, rheumatoid arthritis, and pneumonia caused by Covid-19. Rats were divided into four groups: Control group, ADR group ( $2 \mathrm{mg} / \mathrm{kg}$ Adriamycin, one in three days for 30 days, i.p.), CLQ group (50 $\mathrm{mg} / \mathrm{kg}$ Chloroquine, per day for 30 days, i.p.), ADR+CLQ ( $2 \mathrm{mg} / \mathrm{kg}$ Adriamycin, one in three days for 30 days, i.p. and $50 \mathrm{mg} /$ $\mathrm{kg}$ Chloroquine, per day for 30 days, i.p.). Animals were sacrificed, and liver tissues were extracted for further examinations. Histopathological changes in liver tissues were scored and IL-17 immunostaining was performed to determine the expression levels among experimental groups. Bodyweights in the ADR group decreased significantly compared to the Control group and CLQ group. Furthermore, bodyweight in ADR+CLQ group was significantly higher compared to ADR group. The histopathological score was significantly higher in ADR group when compared to Control and CLQ group while CLQ administrations reduced the damage induced by ADR in the ADR+CLQ group. IL-17 immunoreactivity was considerably increased in the ADR group. On the other hand, IL-17 expressions of ADR+CLQ were substantially less compared to ADR group. We suggest that CLQ can be used as a therapeutic agent to reduce the detrimental effects of ADR, thanks to its anti-inflammatory properties.
\end{abstract}

Keywords: Adriamycin, chloroquine, IL-17, liver damage.

${ }^{1}$ Erciyes University, Science Faculty, Biology

Department, Kayseri, Turkey.

${ }^{2}$ Yozgat Bozok University, Faculty of Medicine, Histology-Embryology Department, Yozgat, Turkey ${ }^{3}$ Harran University, Faculty of Medicine, HistologyEmbryology Department, Sanliurfa, Turkey.

${ }^{4}$ Erciyes University, Faculty of Medicine, HistologyEmbryology Department, Kayseri, Turkey.

${ }^{5}$ Ömer Halisdemir University, Niğde Zübeyde Hanım Vocational School of Health Services, Aged Care Service, Nigde, Turkey.

${ }^{6}$ Cappadocia University, Cappadocia Vocational

School, Department of Medical Services and

Technique, Pathology Laboratory Techniques Pr.,

Nevsehir, Turkey

${ }^{*}$ Corresponding author:

Ali Tuğrul Akin

E-mail address: Tugrul.akinn@gmail.com

Adress: Erciyes University, Science Faculty, Biology

Department, C2-1K-13, Kayseri, Turkey.

Telephone number: +90 5514318549

DOI: 10.2478/ebtj-2021-0003

(๑) 2021 Authors. This work was licensed under the Creative Commons Attribution-NonCommercialNoDerivs 4.0 License.

\section{Introduction}

Cancer, which is a disease characterized by abnormal and uncontrolled proliferation of cells and which results in death in many types of species, is one of the diseases with the highest mortality. For this reason, scientists are conducting extensive research to treat this disease (1).

With the discovery of antineoplastic chemotherapy, significant reductions in mortality and morbidity rates of cancer disease occurred. However, besides the benefits of the drugs used in chemotherapy, the long-term use of these drugs caused serious damage to tissue and organ systems in cancer patients as a result of their cytotoxic effects (2).

Doxorubicin (DOX), an active ingredient of Adriamycin (ADR), is an anthracycline antibiotic class and has been widely used in cancer treatment for more than 30 years. DOX was first obtained from Streptomyces peucetius, a fungus species. As one of the anthracycline antibiotics, ADR is the most widely used drug in the treatment against solid tumors. Moreover, ADR is also widely used in the treatment of many types of cancer, such as bladder and breast cancer (3-6). Although ADR is a drug with strong antineoplastic activity in various cancers, its clinical use is limited due to its negative side effects such as cardiotoxicity (7), nephrotoxicity (8), hepatotoxicity (9) and gonadotoxicity (10).

During the elucidation of the mechanism of ADR toxicity, two main theories have been introduced. One of them states that DOX inhibits the maintenance of the topoisomerase 
II enzyme by intercalating with the DNA double chain and finally suppresses DNA replication and transcription. Another theory is that DOX induces oxidative stress, causing damage to healthy tissues and directing the cell to apoptosis (11). In addition, DOX causes histone separation from active chromosomes, creating a serious DNA damage (12).

Many experimental studies have reported that ADR chemotherapy causes serious hepatotoxicity $(9,13)$. The mechanism of ADR toxicity has been associated with the oxidative stress state associated with the formation of an excessive amount of reactive oxygen species (ROS) and/or a reduction in the antioxidant defense system that causes unbalanced normal oxygen metabolism. First, a form of semi-quinone is produced as a result of adding an electron to the quinone half of DOX, and then the quinone form is rapidly formed by the reduction of molecular oxygen to ROS (14).

The formation of excessive reactive oxygen species (ROS), suppression of the antioxidant defense system and lipid peroxidation of biological membranes cause overexpression of the genes in inflammatory pathways and may cause excessive release of pro- and anti-inflammatory cytokines such as Tumor Necrosis Factor-alpha (TNF- $\alpha)$ and Interleukin-17 (IL-17). Finally, the imbalance between these cytokines can induce necrosis and apoptosis. Cytokines, which are polypeptides produced and secreted by various cell types, regulate immune and inflammatory events, including a systemic response to inflammation, cell growth, healing and injury $(15,16)$.

Interleukin-17A (IL-17) is a founding member of a new proinflammatory cytokine family of IL-17A-17F with at least six members (17). IL-17 has powerful effects on a large number of cells of the innate immune system, especially granulocyte lineage, and is important in bridging adaptive and congenital immune systems (18). Many studies have shown that ADR activates inflammatory pathways and triggers apoptosis in many tissue types, causing overexpression of inflammatory cytokines such as TNF- $\alpha$ and $I L-17$ (19-22).

Chloroquine (CLQ) is a drug used clinically as an anti-inflammatory (23) and it is used in the treatment of many inflammatory diseases such as malaria (24), rheumatoid arthritis (25) and widespread pneumonia caused by Covid-19 virus, which occurred in Wuhan, China at the end of 2019, affecting the whole World (26). The action of TNF- $\alpha$, which induces the expression of other interleukins, is inhibited by the lysosomal protease inhibitor CLQ (27). CLQ therapy has been reported to increase mortality in mice by inhibiting inflammatory pathways and apoptosis (28).

The aim of this study is to investigate the effect of CLQ, an anti-inflammatory drug, on histopathological changes in liver tissue and $I L-17$ expression in ADR-induced liver damage in rats. For this purpose, histopathological examinations were performed to determine the ameliorative effects of CLQ on the damage caused by ADR in the liver, and changes in the IL-17 expression in the liver tissue were determined by immunohistochemical staining.

\section{Material and Methods Experimental design}

In this study, thirty-two male Wistar albino rats (8 weeks old, weighing 100-200 gr) were obtained from Hakan Cetinsaya Experimental and Clinic Research Center, Erciyes University, Kayseri, Turkey. During the experiment, rats were harbored in a $12 \mathrm{~h} \mathrm{light} / 12 \mathrm{~h}$ dark cycle at room temperature $(20-24$ ${ }^{\circ} \mathrm{C}$ ) and environmental humidity. Standard chow and tap water were given to animals ad libitum. At the beginning of the experiment, the rats randomly divided into four groups as follows; The Control group $(n=8)$ were untreated rats, ADR $(\mathrm{n}=8)$ group administered $2 \mathrm{mg} / \mathrm{kg}$ Adriamycin intraperitoneally (i.p) one in three days for 30 days similar to literature (29), CLQ group ( $\mathrm{n}=8$ ) group given $50 \mathrm{mg} / \mathrm{kg}$ Chloroquine for 30 days (30) and ADR plus CLQ group ( $\mathrm{n}=8$ ) given $2 \mathrm{mg} / \mathrm{kg}$ Adriamycin one in three days and $50 \mathrm{mg} / \mathrm{kg}$ Chloroquine for 30 days. After experimental procedure, animals were anesthetized with ketamine and xylazine combination and they were sacrificed after body weights were saved. Liver tissues were extracted from the animals for the histopathological, immunohistochemical examinations.

\section{Histopathological examinations}

Histopathological evaluation of the structure of the liver tissue was performed using routine histological methods. The liver tissues were fixed in 10\% formalin solution for 24-48 h, dehydrated with alcohol, cleared with xylene and embedded in paraffin wax and cut into 5- $\mu \mathrm{m}$ thick sections. Hematoxylin-eosin (H\&E) staining were performed for the evaluation of the histopathological changes in the liver tissue (37-39). Photographs were taken with a photomicroscope (Olympus BX51; Olympus, Tokyo, Japan) and analyzed by the study group. The liver tissue structure was examined and evaluated randomly and with standard light microscopy and were scored by the study group. While applying histopathological score, the following criteria were used; hemorrhage, necrotic hepatocytes, vacuolized hepatocytes and the appearance of hepatocyte cords. Scoring was conducted as follows: $0=$ not at all, $1=0-25 \%, 2=26-45 \%, 3$ $=46-75 \%$, and $4=76-100 \%$.

\section{Immunohistochemistry}

The immunohistochemistry method was applied according to previous studies (31-33) to investigate the changes in the immunoreactivity of $I L-17$ antibody in liver tissues. $5 \mu \mathrm{m}$ sections were obtained from liver tissues embedded in paraffin blocks. The sections were kept in the oven at 60 degrees for at least 2 hours so that the paraffin melted. The tissues were deparaffinized and rehydrated using xylene and alcohol series. Sections were taken into a sterile urine container with $0.01 \mathrm{M}$ citrate buffer and heated in a microwave oven at $350 \mathrm{~W}$ for the antigen retrieval. Then, sections were kept three times in phosphate-buffered saline (PBS) for 5 minutes. The sections were kept in $3 \%(\mathrm{w} / \mathrm{v}) \mathrm{H}_{2} \mathrm{O}_{2}$ for 10 minutes to block endogenous peroxidase activity. After washing again 3 times in PBS (phosphate buffered saline), Ultra V Block solution was added to the tis- 
sues and kept in the tank for 5 minutes. After then, $I L-17$ (Cat. No: bs-1183R, Bioss) antibody diluted in the ratio of 1:100 were added to the tissues and incubated overnight at $4{ }^{\circ} \mathrm{C}$. The following morning, the tissues were washed again three times with PBS and the secondary antibody (TA-125-HDX, Thermo Fisher Scientific, Waltham, MA, USA) was instilled for 10 minutes at room temperature. After washing with PBS, the immunoreaction was amplified using the streptavidin-avidin-peroxidase complex and the sections were visualized using 3,3-p-diaminobenzidine tetrahydrochloride (TA-060-HDX, Thermo Fisher Scientific, Waltham, MA, USA) lightly counter-stained with Gill hematoxylin. For the final step, increasing alcohol serial concentrations were used to remove water, the sections were then passed through xylene, and finally, they were covered with entellan. Density of $I L-17$ immunoreactivity were scored as follows: $0=$ no staining; $1=$ weak immunoreactivity; $2=$ moderate immunoreactivity; $3=$ strong immunoreactivity (40-42).

\section{Statistical analysis}

All statistical analyses were carried out by using GraphPad Prism version 7.00 for Mac, GraphPad Software, La Jolla, California, USA. D’Agostino Pearson omnibus test was used to identify the normal distribution of the data. In the case of normal distribution, quantitative variables were compared using one-way analysis of variance (ANOVA) and Tukey's posthoc test. The data were expressed as the mean of normalized data \pm standard deviation of the mean. $p<0.05$ was considered as statistically significant.

\section{Results \\ Histopathological findings}

Final body weights in the ADR group decreased significant- ly compared to the Control group, CLQ group and the initial body weights of ADR group $(p<0.05)$. Furthermore, body weight in the ADR +CLQ group were significantly higher compared to those of the ADR group $(p<0.05)$ (Figure 1A). Necrotic hepatocytes, vacuolized hepatocytes and hemorrhage were observed in the ADR group and hepatocytes cords were irregular were compared to Control and CLQ group (Fig 2C). In the ADR+CLQ group, hepatocyte cords were relatively regular and necrotic and vacuolized hepatocytes and hemorrhage were less when compared to ADR group (Fig 2D). Moreover, histopathological score was higher in the ADR group when compared to Control and CLQ group. Unlikely, ADR+CLQ group showed relatively less histopathological score when compared to ADR group, suggesting an ameliorative effect of CLQ on the liver damage induced by ADR (Figure 1B).

\section{Immunohistochemical findings}

Immunohistochemical staining was performed by using the avidin-biotin method to determine the liver tissue expressions of $I L-17$. Immunohistochemical examinations demonstrated the presence of $I L-17$ immunoreactivity in the liver tissues of experimental groups. The $I L-17$ expressions in the liver tissues of CLQ group were similar to those in the Control group. Especially, $I L-17$ immunoreactivity was considerably increased in the liver tissue sections of ADR group. On the other hand, IL17 expressions of ADR+CLQ were substantially less compared to those in the ADR group. Figure 3 shows the $I L-17$ expressions and statistical analysis of the score of immunohistochemical staining in experimental groups.

\section{Discussion}

Despite Adriamycin's potent antineoplastic activity in many
$\mathbf{A}$

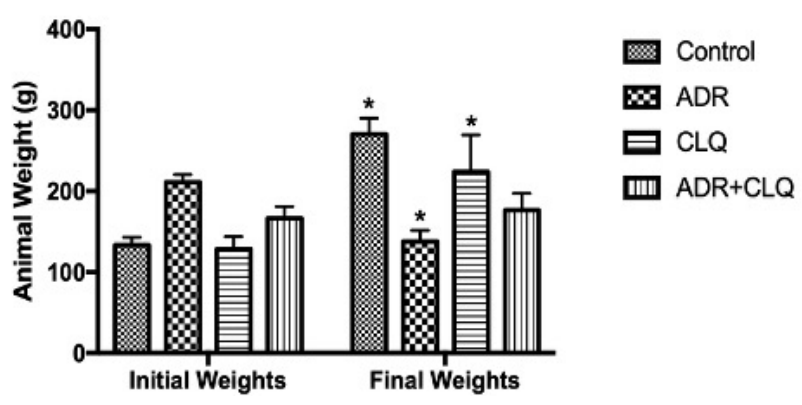

B

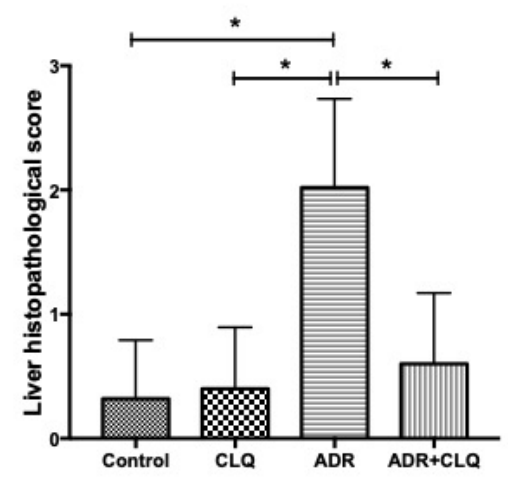

Figure 1. A, B. (A) Changes in the body weights of the experimental animals before and after the application of the experimental procedure. (B) Final body weights in the ADR group decreased significantly compared to the Control group, CLQ group and initial body weights of ADR group $(p<0.05)$. Furthermore, body weight in the ADR+CLQ group were significantly higher compared to those of the ADR group ( $p<0.05)$. Histopathological scores showed a significant increase in the histopathological score in the ADR group when compared to the Control and CLQ group. However, it is also seen a significant decrease in the histopathological score in the ADR+CLQ group when compared to the ADR group. Abbreviations: ADR, Adriamycin; CLQ, Chloroquine. 


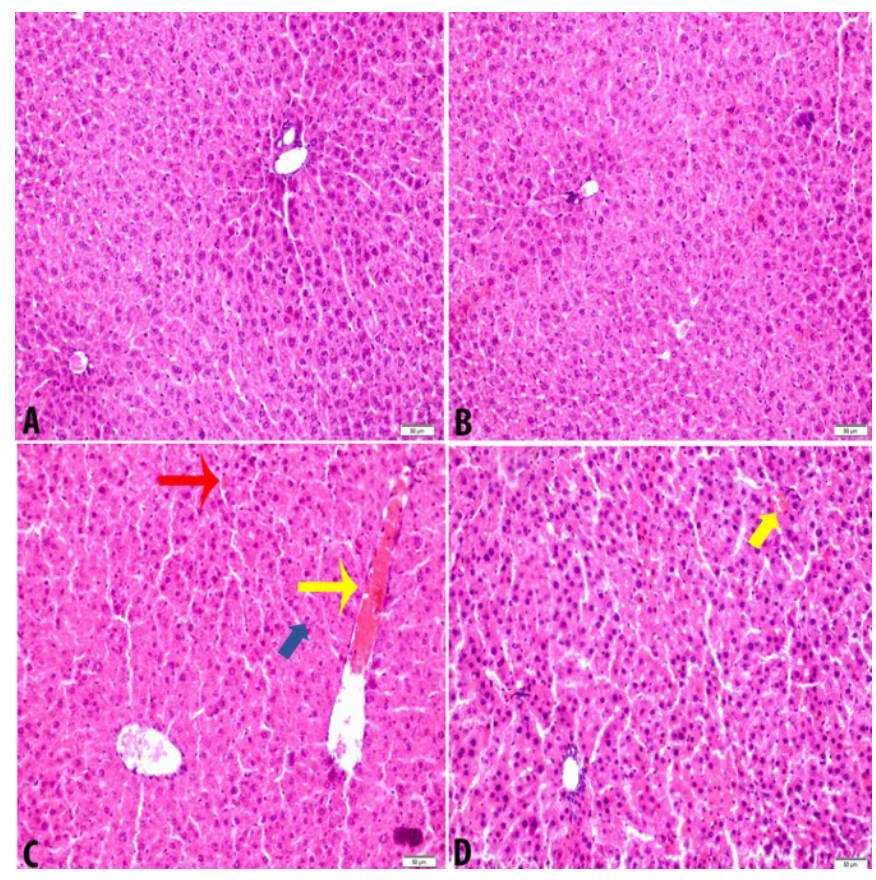

Figure 2. A-D. Light microscopy of liver tissue stained with H\&E in experimental groups. (A) Control group ( $\mathrm{n}=8$ ) and (B) CLQ group ( $\mathrm{n}=8$ ), normal liver were observed; (C) ADR group ( $n=8$ ), hemorrhage (yellow arrow), necrotic hepatocytes (red arrow), vacuolized hepatocytes (blue arrow), and irregular hepatocytes cords were observed; and (D) ADR+CLQ group, near to normal liver tissue were observed except for small hemorrhagic regions (yellow arrow); Scala bar: $50 \mu$. Magnification x200. Abbreviations: ADR, Adriamycin; CLQ, Chloroquine.
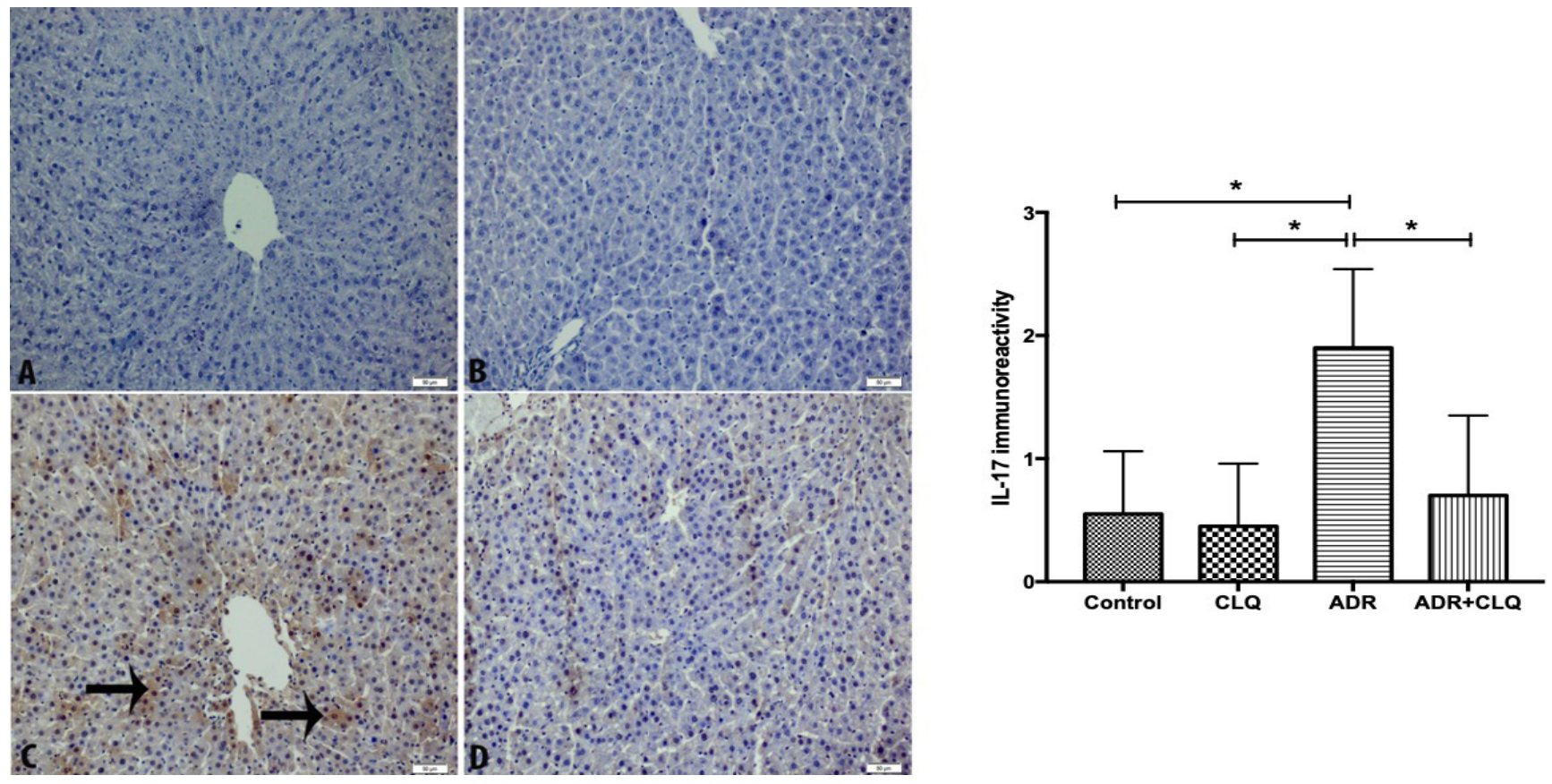

Figure 3. A-D. Immunohistochemical staining of $I L-17$ in the liver tissues and statistical analysis of the score of immunohistochemical staining in the experimental groups. (A) Control $(n=8)$ and (B) CLQ ( $n=8)$ showed weak $I L-17$ immunostaining in the liver tissue sections; (C) ADR $(\mathrm{n}=8)$ group, $I L-17$ expression increased in the liver tissue. (D) ADR+CLQ ( $\mathrm{n}=8) I L-17$ expression was significantly decreased when compared to the ADR group. Arrows show the IL-17 immunopositive cells. Graph shows the statistical analysis of the score of immunohistochemical staining according to the following criteria: 0 , no staining; 1 , weak immunoreactivity; 2 , moderate immunoreactivity; 3 , strong immunoreactivity. Scala bar: $50 \mu$. Magnification x200. Abbreviations: ADR, Adriamycin; CLQ, Chloroquine. 
Table 1. Initial and final body weights, histopathological, and IL-17 immunostaining score among experimental groups.

\begin{tabular}{|c|c|c|c|c|c|c|}
\hline \multicolumn{2}{|c|}{ Groups } & Control & CLQ & ADR & ADR+CLQ & P \\
\hline $\begin{array}{c}\text { Body weight } \\
\text { (gr) }\end{array}$ & Initial weight & $133 \pm 10.2$ & $128.3 \pm 15.6$ & $211.1 \pm 9.4$ & $166.5 \pm 14.5$ & $\mathbf{0 . 0 0 1}$ \\
\hline Final weight & $270.3 \pm 19.8^{\star}$ & $223.7 \pm 45.8^{*}$ & $137.7 \pm 13.7^{*}$ & $176.6 \pm 20.4$ & $\mathbf{0 . 0 0 1}$ \\
\hline Histopathological score (0 to 3) & $0.32 \pm 0.47^{\mathrm{a}}$ & $0.40 \pm 0.49^{\mathrm{a}}$ & $2.02 \pm 0.71^{\mathrm{b}}$ & $0.60 \pm 0.57^{\mathrm{a}}$ & $\mathbf{0 . 0 0 1}$ \\
\hline Score of $I L-17$ staining $(0$ to 3) & $0.55 \pm 0.51^{\mathrm{a}}$ & $0.45 \pm 0.51^{\mathrm{a}}$ & $1.90 \pm 0.64^{\mathrm{b}}$ & $0.70 \pm 0.65^{\mathrm{a}}$ & $\mathbf{0 . 0 0 1}$ \\
\hline
\end{tabular}

Data are expressed as mean \pm standard deviation and $p<0.05$ was considered as significant.

* Significant when compared to initial body weight.

There is no significant difference between the groups with same letter $(a, b)$.

Abbreviations: ADR, Adriamycin; CLQ, Chloroquine; IL-17, Interleukin-17.

types of cancer, its clinical use is limited because of its detrimental side effects in many healthy organs. Many experimental studies have reported that ADR administrations cause a significant decrease in body weights in experimental animals (34). In our study, we used Wistar albino rats as experimental animals and after ADR and CLQ administrations we observed a significant decrease in body weight in the ADR group when compared to its initial body weights and those in the Control group. Moreover, we noticed that ADR administration affected a little bit the weight gain in the ADR +CLQ group when compared to Control group. We think that this decrease in body weight in the ADR group and the inhibition of the weight gain in the ADR +CLQ group may be induced by the damage of ADR on multiple organ systems in the body.

Many experimental studies have reported that ADR chemotherapy causes serious hepatotoxicity by inducing the excessive formation of ROS and the overexpression of the genes involved in inflammatory pathways, and so inhibiting the antioxidant defense system and driving cell to apoptosis $(9,13)$. In this experimental study, we observed that ADR administrations caused significant damage in the liver tissue including hemorrhage, necrotic hepatocytes, vacuolized hepatocytes, and irregular hepatocyte cords when compared to Control and CLQ groups. However, we also noticed that CLQ administrations ameliorated the damage induced by ADR in the liver tissue because necrotic hepatocytes and vacuolized hepatocytes were almost nonexistent in the ADR +CLQ group. Moreover, lobular hemorrhage was less, and hepatocyte cords were more regular when compared to ADR group. According to our histopathological examinations, we suggest that CLQ administrations have significant ameliorative effects in the liver tissue by inhibiting the inflammatory pathways because oxidative stress, inflammation, and apoptosis are closely related events in the many cell types in the body.

Several studies have reported that ADR chemotherapy triggers oxidative stress-related inflammation by inducing the overexpression of the genes regulating the inflammatory events. There are several cytokines and chemokines that regulates many pathways in the inflammatory events. In many experimental studies, it has been shown that ADR administration cause the upregulation of the pro- and anti-inflammatory cytokines such as TNF- $\alpha, I L-6$, and $I L-1 \beta(35,36)$, suggesting that a serious inflammation is triggered by Adriamycin administrations, but changes in the IL-17 expressions in the ADR-induced liver damage has not been clearly elucidated. Our study showed that $I L-17$ expressions were clearly observed in the liver tissue of experimental groups via immunohistochemical staining. According to our immunohistochemical results, $I L-17$ expression significantly increased in only ADR administered group when compared to Control and CLQ groups. However, we also observed that CLQ administrations significantly decreased the IL-17 expressions in the ADR +CLQ group. In the light of these results, we suggest that CLQ administrations may suppress the inflammation by inhibiting the $I L-17$ expressions in the liver tissue. However, changes in the expression of more cytokines and chemokines need to be examined to conclude that CLQ inhibits inflammation in liver tissue and protects the cell from apoptosis and it is the limitation of our study. In addition, IL-17 expression has not been previously demonstrated in ADR-induced liver injury in the literature. In our study, we think that we contribute to the literature by showing the changes in IL-17 expression in ADR-induced liver damage through immunohistochemical staining.

\section{Conclusion}

As a result, we suggest that Chloroquine can be used as an ameliorative agent at low doses for reducing the negative effects of Adriamycin because of its anti-inflammatory properties. Moreover, we think that the outcomes of this study will contribute to the reconsideration of existing treatment methods and the determination of new strategies in Adriamycin-induced liver damage.

\section{Compliance with ethical standards}

The experimental protocol performed in the present study was accepted by the Erciyes University's Experimental Animal and Local Ethics' Committee with number 19/205/2019.

\section{Conflict of interest statement}

The authors declare that they have no conflict of interest.

\section{References}

1. Ölgen S BaI, Nebioğlu D. Angiogenesis and New Aspects of Cancer Chemotherapy. Journal of Faculty of Pharmacy 
of Ankara University. 2002;31 (3):193-214.

2. Meister LA MALeocctCPP, 102-131. Late effects of childhood cancer therapy. Current Problems in Pediatrics 1993 23:102-31.

3. Doroshow JH, Locker GY, Myers CE. Enzymatic defenses of the mouse heart against reactive oxygen metabolites: alterations produced by doxorubicin. J Clin Invest. 1980;65(1):128-35.

4. Kismet E DE, Yurttutan N, Berberoğlu S, Karademir S, Aydın Hİ, Kalman S, Köseoğlu V. Serum BNP levels in children treated with Doxorubicin for their solif tumors. Gülhane Tıp Dergisi 2004;46(1):38-42.

5. Liu Y, Yang Y, Liu X, Jiang T. Quantification of pegylated liposomal doxorubicin and doxorubicinol in rat plasma by liquid chromatography/electrospray tandem mass spectroscopy: Application to preclinical pharmacokinetic studies. Talanta. 2008;74(4):887-95.

6. Wallace K. Doxorubicin-induced cardiac mitochondrionopathy. Pharmacology and Toxicology. 2003;93(3):105-15.

7. Li J, Li L, Li X, Wu S. Long noncoding RNA LINC00339 aggravates doxorubicin-induced cardiomyocyte apoptosis by targeting MiR-484. Biochem Biophys Res Commun. 2018;503(4):3038-43.

8. El-Sayed EM, Mansour AM, El-Sawy WS. Protective effect of proanthocyanidins against doxorubicin-induced nephrotoxicity in rats. J Biochem Mol Toxicol. 2017;31(11).

9. Omobowale TO, Oyagbemi AA, Ajufo UE, Adejumobi OA, Ola-Davies OE, Adedapo AA, et al. Ameliorative Effect of Gallic Acid in Doxorubicin-Induced Hepatotoxicity in Wistar Rats Through Antioxidant Defense System. J Diet Suppl. 2018;15(2):183-96.

10. Hozayen W. Effect of hesperidin and rutin on doxorubicin induced testicular toxicity in male rats. International Journal of Food Sciences and Nutrition. 2012;1:31-42.

11. Fimognari C, Sestili P, Lenzi M, Bucchini A, Cantelli-Forti G, Hrelia P. RNA as a new target for toxic and protective agents. Mutat Res. 2008;648(1-2):15-22.

12. Pang B, Qiao X, Janssen L, Velds A, Groothuis T, Kerkhoven $\mathrm{R}$, et al. Drug-induced histone eviction from open chromatin contributes to the chemotherapeutic effects of doxorubicin. Nat Commun. 2013;4:1908.

13. Bilgic S, Ozgocmen M. The protective effect of misoprostol against doxorubicin induced liver injury. Biotech Histochem. 2019;94(8):583-91.

14. Liu J, Qu W, Kadiiska MB. Role of oxidative stress in cadmium toxicity and carcinogenesis. Toxicol Appl Pharmacol. 2009;238(3):209-14.

15. Noronha IL, Niemir Z, Stein H, Waldherr R. Cytokines and growth factors in renal disease. Nephrol Dial Transplant. 1995;10(6):775-86.

16. Azuma MM, Gomes-Filho JE, Prieto AKC, Samuel RO, de Lima VMF, Sumida DH, et al. Diabetes increases interleukin-17 levels in periapical, hepatic, and renal tissues in rats. Arch Oral Biol. 2017;83:230-5.

17. Aggarwal S, Gurney AL. IL-17: prototype member of an emerging cytokine family. J Leukoc Biol. 2002;71(1):1-8.

18. Yu JJ, Gaffen SL. Interleukin-17: a novel inflammatory cytokine that bridges innate and adaptive immunity. Front Biosci. 2008;13:170-7.

19. He Y, Yang Z, Li J, Li E. Dexmedetomidine reduces the inflammation and apoptosis of doxorubicin-induced myocardial cells. Exp Mol Pathol. 2020;113:104371.

20. Zhang S, You ZQ, Yang L, Li LL, Wu YP, Gu LQ, et al. Protective effect of Shenmai injection on doxorubicin-induced cardiotoxicity via regulation of inflammatory mediators. BMC Complement Altern Med. 2019;19(1):317.

21. Liu Y, Su L, Lin Q, Han Y, You P, Fan Q. Induction of C-Mip by IL-17 Plays an Important Role in Adriamycin-Induced Podocyte Damage. Cell Physiol Biochem. 2015;36(4):1274-90.

22. Ma Y, Aymeric L, Locher C, Mattarollo SR, Delahaye NF, Pereira $\mathrm{P}$, et al. Contribution of IL-17-producing gamma delta $\mathrm{T}$ cells to the efficacy of anticancer chemotherapy. J Exp Med. 2011;208(3):491-503.

23. Ben-Zvi I, Kivity S, Langevitz P, Shoenfeld Y. Hydroxychloroquine: from malaria to autoimmunity. Clin Rev Allergy Immunol. 2012;42(2):145-53.

24. Lalloo DG, Shingadia D, Pasvol G, Chiodini PL, Whitty CJ, Beeching NJ, et al. UK malaria treatment guidelines. J Infect. 2007;54(2):111-21.

25. Rainsford KD, Parke AL, Clifford-Rashotte M, Kean WF. Therapy and pharmacological properties of hydroxychloroquine and chloroquine in treatment of systemic lupus erythematosus, rheumatoid arthritis and related diseases. Inflammopharmacology. 2015;23(5):231-69.

26. Huang M, Tang T, Pang P, Li M, Ma R, Lu J, et al. Treating COVID-19 with Chloroquine. J Mol Cell Biol. 2020;12(4):322-5.

27. Lehmann GL, Carreras FI, Soria LR, Gradilone SA, Marinelli RA. LPS induces the TNF-alpha-mediated downregulation of rat liver aquaporin-8: role in sepsis-associated cholestasis. Am J Physiol Gastrointest Liver Physiol. 2008;294(2):G567-75.

28. Yang M, Cao L, Xie M, Yu Y, Kang R, Yang L, et al. Chloroquine inhibits HMGB1 inflammatory signaling and protects mice from lethal sepsis. Biochem Pharmacol. 2013;86(3):410-8.

29. Shivakumar P, Rani MU, Reddy AG, Anjaneyulu Y. A study on the toxic effects of Doxorubicin on the histology of certain organs. Toxicol Int. 2012;19(3):241-4.

30. Long L, Yang X, Southwood M, Lu J, Marciniak SJ, Dunmore BJ, et al. Chloroquine prevents progression of experimental pulmonary hypertension via inhibition of autophagy and lysosomal bone morphogenetic protein type II receptor degradation. Circ Res. 2013;112(8):1159-70.

31. Bayatli F, Akkus D, Kilic E, Saraymen R, Sonmez MF. The protective effects of grape seed extract on MDA, AOPP, apoptosis and eNOS expression in testicular torsion: an experimental study. World J Urol. 2013;31(3):615-22.

32. Karabulut D, Ulusoy HB, Kaymak E, Sönmez MF. Thera- 
peutic effects of pentoxifylline on diabetic heart tissue via NOS. Anatolian journal of cardiology. 2016;16(5):310-5.

33. Öztürk E, Kaymak E, Akin AT, Karabulut D, Ünsal HM, Yakan B. Thymoquinone is a protective agent that reduces the negative effects of doxorubicin in rat testis. Hum Exp Toxicol. 2020:960327120924108.

34. Afsar T, Razak S, Almajwal A. Effect of Acacia hydaspica R. Parker extract on lipid peroxidation, antioxidant status, liver function test and histopathology in doxorubicin treated rats. Lipids in Health and Disease. 2019;18(1):126.

35. Kuzu M, Yıldırım S, Kandemir FM, Küçükler S, Çağlayan C, Türk E, et al. Protective effect of morin on doxorubicin-induced hepatorenal toxicity in rats. Chem Biol Interact. 2019;308:89-100.

36. Song S, Chu L, Liang H, Chen J, Liang J, Huang Z, et al. Protective Effects of Dioscin Against Doxorubicin-Induced Hepatotoxicity Via Regulation of Sirt1/FOXO1/NF кb Signal. Front Pharmacol. 2019;10:1030.

37. Doğanyiğit Z, Yakan B, Okan A, Silici S. Antioxidative role of propolis on LPS induced renal damage. The EuroBiotech Journal. 2020;4:156-60.

38. Karabulut D, Ozturk E, Kuloglu N, Akin AT, Kaymak E, Yakan B. Effects of vitamin B12 on methotrexate hepatotoxicity: evaluation of receptor-interacting protein (RIP) kinase. Naunyn Schmiedebergs Arch Pharmacol. 2020;393(12):2473-80.

39. Akin A. T. KE, Karabulut D. , Doğanyiğit Z., Ceylan T., Toluk A. , Özdamar S. Comparison of the acute and cumulative dose administrations in doxorubicin-induced hepatotoxicity via evaluation of the histopathological changes and inflammation in rats. Experimental and Applied Medical Science. 2020;1(3):73-81.

40. Doğanyiğit Z, Silici S, Demirtaş A, Kaya E, 1. Kaymak E. Determination of histological, immunohistochemical and biochemical effects of acute and chronic grayanotoxin III administration in different doses in rats. Environ Sci Pollut Res Int. 2019;26(2):1323-35.

41. Çakır M, Tekin S, Doğanyiğit Z, Çakan P, Kaymak E. The protective effect of cannabinoid type 2 receptor activation on renal ischemia-reperfusion injury. Mol Cell Biochem. 2019;462(1-2):123-32.

42. Sönmez MF, Ozdemir Ş, Guzel M, Kaymak E. The ameliorative effects of vinpocetine on apoptosis and HSP-70 expression in testicular torsion in rats. Biotech Histochem. 2017;92(2):92-9. 